\title{
GVHD-free, relapse-free survival provides novel clues for optimizing allogeneic-HSCT for adult T-cell leukemia/lymphoma
}

\author{
Hiroyuki Muranushi ${ }^{1}$ Takero Shindo ${ }^{1}{ }^{1} \cdot$ Masakatsu Hishizawa ${ }^{1}$ Masahito Tokunaga ${ }^{2} \cdot$ Atsushi Wake $^{3}$. \\ Nobuaki Nakano ${ }^{2}$. Tetsuya Eto ${ }^{4}$. Michihiro Hidaka ${ }^{5}$ - Ilseung Choi ${ }^{6} \cdot$ Toshihiro Miyamoto $^{7}$. Naoyuki Uchida ${ }^{8}$. \\ Yukiyoshi Moriuchi $^{9}$ - Yasuhiko Miyazaki ${ }^{10}$. Takahiro Fukuda ${ }^{11}$. Tatsuo Ichinohe ${ }^{12}$. Yoshiko Atsuta ${ }^{13,14} \cdot$ The ATL \\ Working Group of the Japanese Society for Hematopoietic Cell Transplantation • Koji Kato ${ }^{7}$
}

Received: 11 January 2020 / Revised: 11 June 2020 / Accepted: 2 July 2020 / Published online: 14 July 2020

(c) The Author(s), under exclusive licence to Springer Nature Limited 2020

\begin{abstract}
The outcome of allogeneic hematopoietic stem cell transplantation (allo-HSCT) for adult T-cell leukemia/lymphoma (ATL) is still unsatisfactory. To illustrate the advantages and disadvantages of each donor source, we performed a nationwide retrospective study of graft-versus-host disease (GVHD)-free, relapse-free survival (GRFS) of patients with allo-HSCTtreated ATL. One-year GRFS did not significantly differ between patients who received related bone marrow transplantation (R-BMT; 26\%, $n=117$ ), related peripheral blood stem cell transplantation (R-PBSCT; 22\%, $n=225$ ), unrelated bone marrow transplantation (UR-BMT; 26\%, $n=619$ ), and cord blood transplantation (CBT; $21 \%, n=359 ; p=0.09$ ). This was attributable to a low incidence of systemically-treated chronic GVHD after CBT (9\% at 1 year) and reduced non-GVHD/ relapse mortality after R-PBSCT ( $9 \%$ at 1 year). Among patients transplanted in complete remission (CR), 1-year overall survival after $\mathrm{CBT}(52 \%, n=132)$ was not inferior to that after R-BMT $(55 \%, n=51)$, R-PBSCT $(57 \%, n=79)$, and URBMT $(58 \%, n=280 ; p=0.15)$, and relapse rates were equivalent among the four sources $(p=0.19)$. Our results suggest that all donor sources are feasible for CR patients and that GRFS provides important clues toward optimizing allo-HSCT for ATL.
\end{abstract}

Members of the ATL Working Group of the Japanese Society for Hematopoietic Cell Transplantation are listed below Acknowledgements

Supplementary information The online version of this article (https:// doi.org/10.1038/s41409-020-00996-y) contains supplementary material, which is available to authorized users.

Takero Shindo

takeros@kuhp.kyoto-u.ac.jp

1 Department of Hematology/Oncology, Kyoto University Graduate School of Medicine, Kyoto, Japan

2 Department of Hematology, Imamura General Hospital, Kagoshima, Japan

3 Department of Hematology, Toranomon Hospital Kajigaya, Kawasaki, Japan

4 Department of Hematology, Hamanomachi Hospital, Fukuoka, Japan

5 Department of Hematology, Kumamoto Medical Center, Kumamoto, Japan

6 Department of Hematology, National Kyushu Cancer Center, Fukuoka, Japan

\section{Introduction}

Adult T-cell leukemia/lymphoma (ATL) is an aggressive Tcell neoplasm caused by human T-lymphotropic virus type 1 [1] and has a poor prognosis [2-4]. Intensive

7 Department of Medicine and Biosystemic Science, Kyushu University Graduate School of Medical Science, Fukuoka, Japan

8 Department of Hematology, Toranomon Hospital, Tokyo, Japan

9 Department of Hematology, Sasebo City General Hospital, Sasebo, Japan

10 Department of Hematology, Oita Prefectural Hospital, Oita, Japan

11 Department of Stem Cell Transplantation, National Cancer Center Hospital, Tokyo, Japan

12 Research Institute for Radiation Biology and Medicine, Department of Hematology and Oncology, Hiroshima University, Hiroshima, Japan

13 Japanese Data Center for Hematopoietic Cell Transplantation, Nagoya, Japan

14 Nagoya University Graduate School of Medicine, Nagoya, Japan 
chemotherapy and autologous stem cell transplantation have definite limitations [5-9]. Although allogeneic hematopoietic stem cell transplantation (allo-HSCT) may provide long-term remission [10-13], the 3-year survival rate after this procedure is only $33-36 \%$ in Japan $[14,15]$, and similar outcomes have been reported in Europe [16]. Given that grade I-II acute graft-versus-host disease (GVHD) is associated with better overall survival (OS) [17-19], graftversus-leukemia (GVL) effects induced by donor lymphocytes are important in ATL [20, 21]. Mild to moderate GVHD is therefore likely to be beneficial in ATL; however, severe GVHD is associated with non-relapse mortality [17]. Thus, it is difficult to optimize the balance between GVHD and GVL effects while avoiding infection in patients who undergo allo-HSCT for ATL.

Efforts to reduce GVHD decreased transplant-related morbidity/mortality but increased risk of relapse, which may be attributable to diminished GVL effects [22]. On the other hand, efforts to reduce relapse-related mortality by delivering enhanced high-dose chemotherapy prior to alloHSCT led to excess deaths due to organ damage, infections, and GVHD [23]. The final goal of allo-HSCT is to avoid relapse, severe GVHD, and other fatal complications. Therefore, GVHD-free, relapse-free survival (GRFS), defined as the absence of grade III-IV acute GVHD, chronic GVHD that requires systemic treatment, relapse, and death, has been proposed as a novel and clinically meaningful endpoint of allo-HSCT [24]. Cord blood transplantation (CBT) reportedly provides favorable GRFS compared with transplantation from other donor sources in some Japanese populations [25]. The median age of ATL patients is higher and the incidence of fatal opportunistic infection in ATL patients is higher than in patients with other hematologic malignancies [26]. Therefore, we hypothesized that GRFS of ATL patients may differ from that of patients with other hematologic malignancies.

As most ATL patients are over 60 years old, many will not have human leukocyte antigen (HLA)-matched healthy siblings of appropriate age to be eligible as donors. Therefore, alternative donor sources are often utilized in ATL. Among several donor sources, CBT is often performed, especially in patients with relapsing or refractory ATL [27]. This is because such cases require rapid transplantations and the availability of cord blood has recently improved in Japan [28]. HLA-haploidentical transplantation (haploHSCT) is also available. However, such transplants are associated with transplant-related mortality and limited quality of life (QOL) [29]. OS of ATL patients who underwent CBT between 1996 and 2005 was inferior to that of ATL patients who underwent transplantation from other donor sources [14]. Beneficial outcomes of CBT for chemotherapy-sensitive ATL are reported to be feasible $[30,31]$, although these findings should be verified in a larger study. Furthermore, CBT results in a low incidence of GVHD [32], which raises concerns about diminished GVL effects and higher relapse rates. Given that the outcome of CBT for other hematologic malignancies improved after 2006 [33], the limitations and advantages of CBT for ATL should be reevaluated.

Here, we conducted a nationwide retrospective study focusing on OS and GRFS of patients with allo-HSCTtreated ATL after 2006 and compared GRFS among patients who underwent transplantation from different donor sources. Our results illustrate several important advantages and disadvantages of transplantation from each donor source, which may help to improve the overall outcome of allo-HSCT for ATL.

\section{Methods}

\section{Data collection}

Data of 1363 patients with ATL who first underwent alloHSCT between 2006 and 2015 in Japan were retrospectively analyzed. Allo-HSCT recipient clinical data were collected by the Japan Society for Hematopoietic Cell Transplantation (JSHCT) using the Transplant Registry Unified Management Program (TRUMP) [34, 35]. This analysis included the following clinical characteristics of the patients: age at transplantation, gender, disease status at transplantation, date of transplantation, duration from diagnosis to transplantation, and conditioning regimens. The numbers of HLA antigen mismatches in CBT, related bone marrow transplantation (R-BMT), and related peripheral blood stem cell transplantation (R-PBSCT) were counted with respect to $H L A-A,-B$, and $-D R B 1$. HaploHSCT was defined as transplantation with two to three HLA mismatches in the graft-versus-host direction. The number of HLA allelic mismatches in unrelated bone marrow transplantation (UR-BMT) was counted with respect to $H L A-A,-B,-C$, and $-D R B 1$. The present study was approved by the data management committees of the JSHCT as well as the Institutional Review Board of the Kyoto University Graduate School of Medicine. All patients provided consent to participate in the present study.

\section{Definition of endpoints}

OS was defined as the duration from transplantation to death, and the patients who remained alive at the final follow-up were censored. GRFS was defined as the duration from transplantation until death, relapse, development of grade III-IV acute GVHD, or development of chronic GVHD that required systemic treatment, and the patients without any of these events at the time of the final follow-up 
were censored. The relapse date of patients who did not achieve complete remission (CR) before and after transplantation was defined as day 0.1 . Non-GVHD/relapse mortality (NGRM) was defined as the incidence of deaths without relapse, development of grade III-IV acute GVHD, or development of chronic GVHD that required systemic treatment. Patients were divided into two groups according to the conditioning regimens: myeloablative conditioning (MAC) and reduced intensity conditioning (RIC). MAC and RIC were defined according to the proposals of Giralt et al. [36] and Bacigalupo et al. [37] respectively.

\section{Statistical analysis}

Descriptive statistics were used to summarize variables related to the demographics and clinical characteristics of the patients. Groups were compared using Fisher's exact test as appropriate for categorical variables and the Kruskal-Wallis test for continuous variables. The Mann-Whitney $U$ test was used as a nonparametric test to compare two groups. The probabilities of OS and GRFS were estimated according to the Kaplan-Meier method, and univariable comparisons among the groups were performed using the log-rank test. The Cox proportional hazard model was used for multivariate analysis of OS and GRFS. The cumulative incidence rates of relapse and GVHD were estimated, and death without these events was considered as a competing factor. NGRM was also estimated, and its competing events were relapse, grade III-IV acute GVHD, and systemically-treated chronic GVHD. Results were expressed as hazard ratios and their $95 \%$ confidence intervals (CI). All tests were two-sided, and a $p$ value of $<0.05$ was considered to indicate statistical significance. All statistical analyses were performed using Stata (version 13.0, Stata Corporation) and EZR (Saitama Medical Center, Jichi Medical University), a graphical user interface for $\mathrm{R}$ (The $\mathrm{R}$ Foundation for Statistical Computing, version 2.3.0) [38]. The shared scripts from the TRUMP data were used in the analyses [39].

\section{Results}

\section{Characteristics of the patients}

The median patient age was 57 years (range: $20-78$ years) and the median observation period of survivors was 3.1 years (range: $0.0-10.5$ years). The number of CBTs performed in 2011-2015 $(n=240)$ was double the number performed in 2006-2010 $(n=119, p<0.001)$. The median age of patients who underwent CBT was 59 years, which was higher than that of patients who underwent the other types of transplantation $(p<0.001)$. The proportion of patients in $\mathrm{CR}$ at transplantation was lower among those who underwent haplo-HSCT (25\%), CBT (37\%), and RPBSCT $(35 \%)$ than among those who underwent R-BMT $(44 \%)$ and UR-BMT $(45 \% ; p=0.023)$. The duration from diagnosis to transplantation was longer for patients who underwent UR-BMT than for patients who underwent the other types of transplantation $(p<0.001)$. The percentage of HLA-mismatched transplantations was lower among patients who underwent R-BMT (15\%) and higher among patients who underwent CBT $(96 \% ; p<0.001)$. These characteristics are summarized in Table 1. Details of conditioning regimens are summarized in Supplementary Table 1. Of the patients who received MAC, 55\% received cyclophosphamide and total body irradiation, and 13\% received busulfan and cyclophosphamide. Of the patients who received RIC, 55\% received melphalan-based conditioning and 39\% received busulfan-based conditioning.

\section{OS}

First, we compared R-BMT, R-PBSCT, UR-BMT, and CBT. In this analysis, we excluded patients who received haplo-HSCT as there were many fewer than those who received the other transplantation types. One-year OS was worse after CBT $(38 \%, 95 \%$ CI: 32-43\%) than after RBMT (49\%, 95\% CI: 40-58\%), R-PBSCT (52\%, 95\% CI: 45-58\%), and UR-BMT (47\%, 95\% CI: 43-51\%; $p<$ 0.001; Fig. 1a). However, among patients in CR at transplantation, 1-year OS after CBT (52\%, 95\% CI: 43-60\%, $n=132)$ was equivalent to that after R-BMT $(55 \%, 95 \%$ CI: $41-68 \%, n=51)$, R-PBSCT (57\%, 95\% CI: $45-67 \%, n=$ 79), and UR-BMT (58\%, 95\% CI: 52-63\%, $n=280 ; p=$ 0.15; Fig. 1b). On the other hand, in the non-CR patients, 1-year OS after CBT (28\%, 95\% CI: $22-34 \%, n=223)$ was inferior to that after R-BMT ( $45 \%, 95 \%$ CI: $33-57 \%, n=63)$, R-PBSCT (49\%, 95\% CI: 41-57\%, $n=147)$, and UR-BMT (39\%, 95\% CI: $34-44 \%, n=337$; $p<0.001$; Fig. 1c).

\section{Relapse}

The 1-year cumulative incidence of relapse after CBT was 47\% (95\% CI: 42-52\%), which was higher than that after R-BMT (40\%, 95\% CI: 31-49\%), R-PBSCT (42\%, 95\% CI: 35-48\%), and UR-BMT (35\%, 95\% CI: 31-39\%; $p=$ 0.003; Fig. 1d). Among patients in $\mathrm{CR}$ at transplantation, the 1-year cumulative incidence of relapse after CBT (26\%, 95\% CI: 19-34\%) was not significantly different from that after R-BMT (31\%, 95\% CI: 18-44\%), R-PBSCT (31\%, 95\% CI: $21-42 \%)$, and UR-BMT (21\%, 95\% CI: $16-26 \%$; $p=0.19$; Fig. 1e). On the other hand, among the patients in non-CR at transplantation, the 1-year cumulative incidence of relapse after CBT $(60 \%, 95 \%$ CI: 53-66\%) was higher than that after R-BMT (48\%, 95\% CI: 35-60\%), R-PBSCT 
Table 1 Characteristics of the patients.

\begin{tabular}{|c|c|c|c|c|c|c|}
\hline & $\begin{array}{l}\text { R-BMT } \\
N=117\end{array}$ & $\begin{array}{l}\text { R-PBSCT } \\
N=228\end{array}$ & $\begin{array}{l}\text { UR-BMT } \\
N=619\end{array}$ & $\begin{array}{l}\text { CBT } \\
N=359\end{array}$ & $\begin{array}{l}\text { Haplo } \\
N=40\end{array}$ & $P$ \\
\hline Years & & & & & & $<0.001$ \\
\hline 2006-2010 & $66(56)$ & $123(54)$ & $277(45)$ & $119(33)$ & $15(38)$ & \\
\hline 2011-2015 & $51(44)$ & $105(46)$ & $342(55)$ & $240(67)$ & $25(62)$ & \\
\hline Age, median (range) & $53(20-70)$ & $55(25-71)$ & $57(27-76)$ & $59(24-78)$ & $58(26-67)$ & $<0.001$ \\
\hline \multicolumn{7}{|l|}{ Sex } \\
\hline Male & $61(52)$ & $111(49)$ & $355(54)$ & $206(57)$ & $20(50)$ & 0.482 \\
\hline Female & $56(48)$ & $117(51)$ & $283(46)$ & $153(43)$ & $20(50)$ & \\
\hline \multicolumn{7}{|l|}{ Disease status } \\
\hline $\mathrm{CR}$ & $51(44)$ & $79(35)$ & $280(45)$ & $132(37)$ & $10(25)$ & 0.029 \\
\hline Non-CR & $63(54)$ & $147(64)$ & $337(54)$ & $223(62)$ & $29(73)$ & \\
\hline Missing & $3(2)$ & $2(1)$ & $2(0)$ & $4(1)$ & $1(2)$ & \\
\hline \multicolumn{7}{|l|}{ PS } \\
\hline $0-1$ & $102(87)$ & $185(81)$ & $555(90)$ & $306(85)$ & $29(73)$ & 0.029 \\
\hline $2-4$ & $14(12)$ & $41(18)$ & $63(10)$ & $49(14)$ & $10(25)$ & \\
\hline Missing & $1(1)$ & $2(1)$ & $1(0)$ & $4(1)$ & $1(3)$ & \\
\hline \multicolumn{7}{|l|}{ Dx to Trans } \\
\hline$<90$ & $5(4)$ & $30(13)$ & $2(0)$ & $43(12)$ & $4(10)$ & $<0.001$ \\
\hline $90-180$ & $65(56)$ & $101(44)$ & $197(32)$ & $164(46)$ & $16(40)$ & \\
\hline$>180$ & $46(39)$ & $95(42)$ & $419(68)$ & $151(42)$ & $20(50)$ & \\
\hline Missing & $1(1)$ & $2(1)$ & $1(0)$ & $1(0)$ & $0(0)$ & \\
\hline \multicolumn{7}{|l|}{ HLA } \\
\hline Matched & $100(85)$ & $183(80)$ & $418(68)$ & $15(4)$ & $0(0)$ & $<0.001$ \\
\hline Mismatched & $17(15)$ & $45(20)$ & $201(32)$ & $344(96)$ & $40(0)$ & \\
\hline \multicolumn{7}{|l|}{ Conditioning } \\
\hline MAC & $56(48)$ & $91(38)$ & $236(38)$ & $142(40)$ & $10(25)$ & 0.215 \\
\hline RIC & $59(50)$ & $135(62)$ & $382(62)$ & $216(60)$ & $30(75)$ & \\
\hline Missing & $2(2)$ & $2(1)$ & $1(0)$ & $1(0)$ & $0(0)$ & \\
\hline \multicolumn{7}{|l|}{ ATG } \\
\hline Yes & $4(4)$ & $15(7)$ & $17(3)$ & $8(2)$ & $18(45)$ & $<0.001$ \\
\hline No & $113(96)$ & $213(93)$ & 602 (97) & $351(98)$ & $22(55)$ & \\
\hline
\end{tabular}

Data are presented as numbers and (percentages).

$R-B M T$ related bone marrow transplantation, $R-P B S C T$ related peripheral blood stem cell transplantation, $U R-B M T$ unrelated bone marrow transplantation, $C B T$ cord blood transplantation, Haplo haploidentical transplantation, $C R$ complete remission, $P S$ performance status, Dx to Trans Days from diagnosis to transplantation, HLA human leukocyte antigen, MAC myeloablative conditioning, RIC reduced intensity conditioning, ATG anti-thymocyte globulin.
(47\%, 95\% CI: 39-55\%), and UR-BMT (46\%, 95\% CI: $41-52 \% ; p=0.002$; Fig. 1f).

\section{GRFS}

One-year GRFS did not significantly differ after CBT $(21 \%$, 95\% CI: 17-26\%), R-BMT (26\%, 95\% CI: 18-34\%), R-PBSCT (22\%, 95\% CI: 16-27\%), and UR-BMT (26\%, 95\% CI: 22-29\%; $p=0.09$; Fig. 2a). Among patients in CR at transplantation, GRFS did not differ among the four donor sources (CBT: 28\%, 95\% CI: 21-36\%, R-BMT: 35\%, 95\% CI: 22-49\%, R-PBSCT: $27 \%$, 95\% CI: $18-37 \%$, and UR-BMT: $34 \%$, 95\% CI: $29-40 \%$ at 1 year; $p=0.49$; Fig. 2b). Similarly, in the non-CR patients, GRFS did not differ among donor sources at 1 year (CBT: 16\%, 95\% CI: 12-22\%, R-BMT: 19\%, 95\% CI: 11-30\%, R-PBSCT: $18 \%$, 95\% CI: 13-25\%, and UR-BMT: $19 \%$, 95\% CI: 15-23\%; $p=0.25$; Fig. 2c).

We also analyzed the first GRFS events in all the patients and those in CR at transplantation. The rate of NGRM after R-PBSCT $(15 \%)$ was lower than that after the other transplantation types $(26 \%)(p<0.001)$, whereas the incidence of chronic GVHD that required systemic treatment after CBT $(8 \%)$ was lower than that after the other transplantation 
Fig. 1 OS and cumulative incidence of relapse in ATL patients. Kaplan-Meier curves of OS of all patients who received R-BMT, R-PBSCT, UR-BMT, and CBT (a). OS of patients in CR (b) and non-CR (c) at transplantation.

Cumulative incidence of relapse in all patients (d), patients in CR at transplantation (e), and non$\mathrm{CR}$ at transplantation (f). a

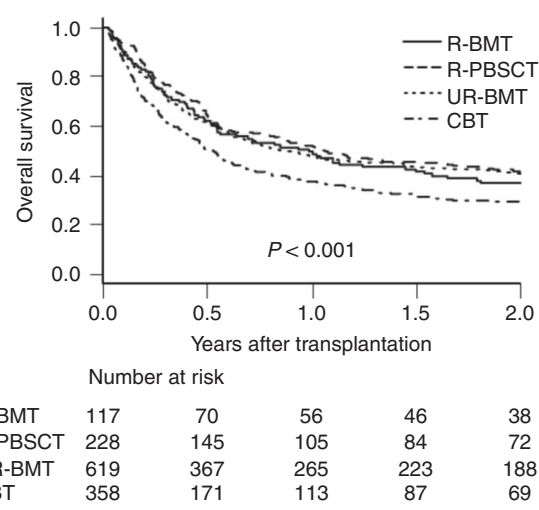

C

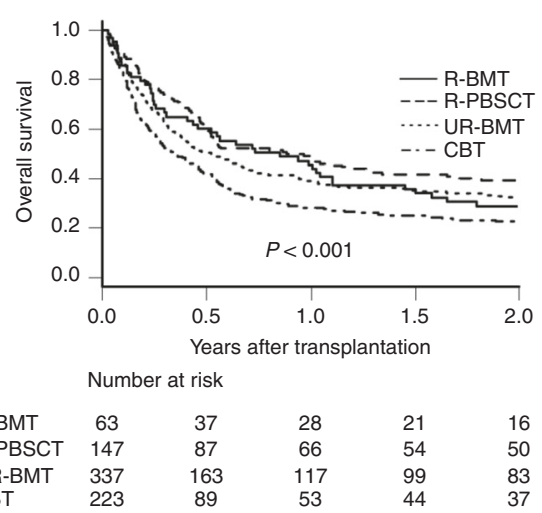

e

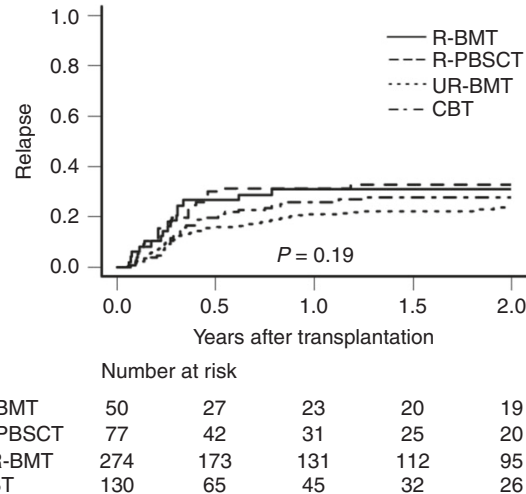

b

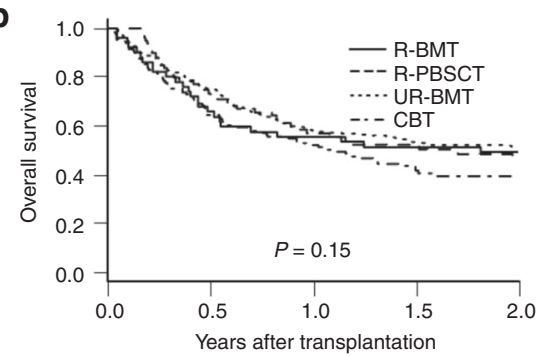
Number at risk

$\begin{array}{lllll}\text { R-BMT } & 51 & 32 & 27 & 24\end{array}$

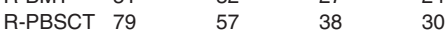

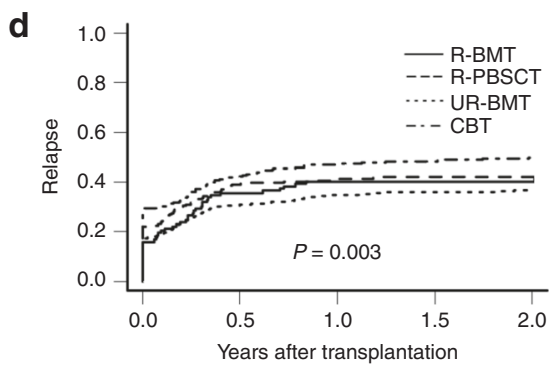
Number at risk

$\begin{array}{lccccc}\text { R-BMT } & 113 & 53 & 45 & 38 & 33 \\ \text { R-PBSCT } & 222 & 100 & 81 & 72 & 66 \\ \text { UR-BMT } & 611 & 297 & 227 & 193 & 167 \\ \text { CBT } & 353 & 126 & 82 & 65 & 52\end{array}$

f

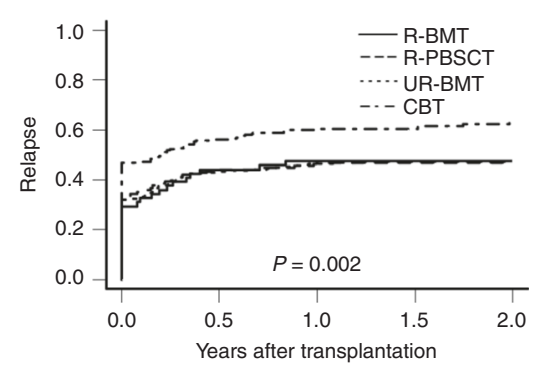
Number at risk

$\begin{array}{lllll}\text { R-BMT } & 61 & 25 & 22 & 18\end{array}$ R-PBSCT 145 $\begin{array}{llr}\text { CBT } & 335 & 124 \\ & 220 & 59\end{array}$ types $(19 \%, p<0.001$; Fig. 2d). Among patients in CR at transplantation, the proportion relapsing after CBT (29\%) was similar to that after the other transplantation types (29\%, $p=0.90)$, but CBT resulted in a higher rate of NGRM $(41 \%)$ compared with the other transplantation types $(28 \%, p=0.014$; Fig. 2e).

\section{GVHD and NGRM}

The 1-year cumulative incidence of grade III-IV acute GVHD after CBT was 11\% (95\% CI: 8-15\%), which was lower than that after R-PBSCT $(20 \%, 95 \%$ CI: $15-26 \%)$ and tended to be lower than that after R-BMT $(17 \%, 95 \% \mathrm{CI}$ : $10-24 \%)$ and UR-BMT $(13 \%, 95 \%$ CI: $11-16 \% ; p=0.014$; Fig. 3a). The percentage of patients with grade II acute
GVHD after UR-BMT was 33\%, which was higher than that after transplantation from the other donor sources (R-BMT: 23\%, R-PBSCT: $24 \%$, and CBT: $20 \%, p<0.001)$. The percentage of patients with chronic GVHD that required systemic therapy at 1 year after CBT was 9\% (95\% CI: 6-12\%), which was lower than that after transplantation from the other sources (R-BMT: 21\%, 95\% CI: 14-29\%, R-PBSCT: $29 \%$, 95\% CI: $23-35 \%$, and UR-BMT: $18 \%$, 95\% CI: 15-21\%, $p<0.001$; Fig. 3b). The 1-year NGRM after R-PBSCT was 9\% (95\% CI: 5-13\%), which was lower than that after transplantation from the other sources (CBT: 20\%, 95\% CI: 16-24\%, R-BMT: 12\%, 95\% CI: 7-19\%, and UR-BMT: 16\%, 95\% CI: 13-19\%; $p<0.001$; Fig. 3c). Similar differences in the incidence of GVHD and NGRM were detected in both the $\mathrm{CR}$ and non-CR patients for each donor source. 
Fig. 2 Comparison of GRFS and first GRFS events among donor sources. Kaplan-Meier curves of GRFS of all patients (a), patients in CR at transplantation (b) and non-CR at transplantation (c). Band graphs showing the percentages of first GRFS events in all patients (d) and patients in CR at transplantation (e).

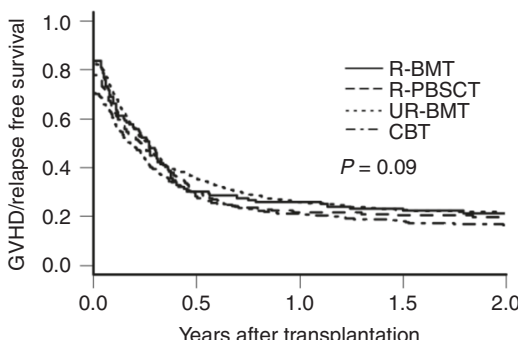

Number at risk

$\begin{array}{lccccc}\text { R-BMT } & 116 & 34 & 29 & 25 & 21 \\ \text { R-PBSCT } & 225 & 63 & 45 & 38 & 34 \\ \text { UR-BMT } & 619 & 215 & 147 & 120 & 98 \\ \text { CBT } & 356 & 102 & 65 & 50 & 39\end{array}$

C

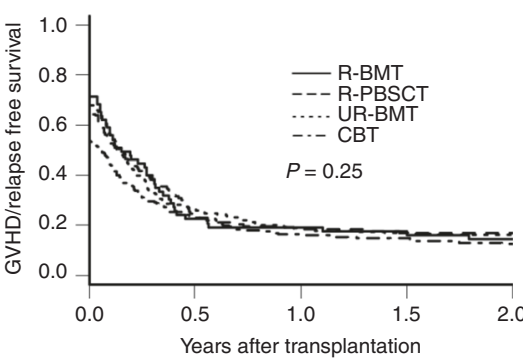

Number at risk

$\begin{array}{lccccc}\text { R-BMT } & 63 & 14 & 12 & 11 & 8 \\ \text { R-PBSCT } & 146 & 35 & 26 & 23 & 23 \\ \text { UR-BMT } & 337 & 86 & 57 & 47 & 38 \\ \text { CBT } & 222 & 49 & 30 & 25 & 20\end{array}$

d

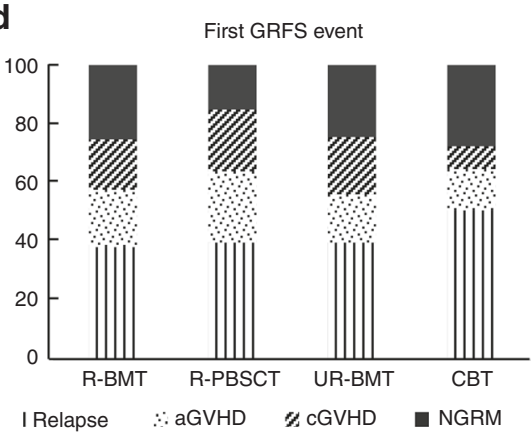

b

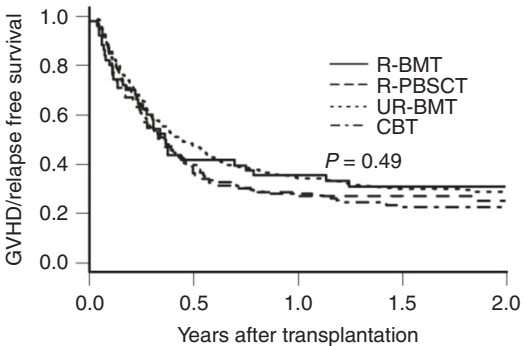

Number at risk

$\begin{array}{lccccc}\text { R-BMT } & 51 & 20 & 17 & 14 & 13 \\ \text { R-PBSCT } & 79 & 28 & 19 & 15 & 11 \\ \text { UR-BMT } & 280 & 129 & 90 & 73 & 60 \\ \text { CBT } & 131 & 51 & 33 & 23 & 19\end{array}$

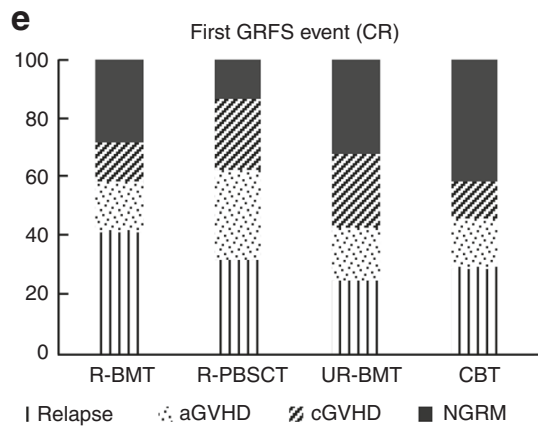

Causes of NGRM are summarized in Table 2. The proportion of deaths caused by infection was significantly higher after CBT $(50 \%)$ than after the other transplantation types $(29 \%, p=0.004)$. This result was associated with a higher incidence of graft failure after CBT (18\%) than after the other transplantation types $(7 \%, p<0.001)$.

\section{HLA matching status}

Next, we compared the outcomes of HLA-matched transplantation with HLA-mismatched (including HLA-haploidentical) transplantation. OS, relapse, grade III-IV acute GVHD, chronic GVHD that required systemic treatment, NGRM, and GRFS did not significantly differ between patients who underwent HLA-matched BMT and those who underwent HLA-mismatched BMT (data not shown).
PBSCT was divided into three groups: HLA 6/6-matched, 5/6-matched, and haploidentical in the graft-versus-host direction. The proportion of CR patients in each group was $35 \%, 29 \%$, and $30 \%$, respectively. For GVHD prophylaxis, $67 \%$ of the patients in the HLA 5/6-matched transplantation group received standard prophylaxis (calcineurin inhibitor plus methotrexate or mycophenolate mofetil), while $24 \%$ received anti-thymocyte globulin (ATG) and $4 \%$ posttransplant cyclophosphamide (PTCY), in addition to standard prophylaxis. In the haplo-HSCT group, $47 \%$ of patients received ATG, 25\% PTCY, and 25\% corticosteroids, in addition to standard prophylaxis. One-year OS after HLA-haploidentical PBSCT was 39\% (95\% CI: 23-55\%), which tended to be inferior to that after HLA 6/6-matched (53\%, 95\% CI: 45-60\%) and HLA 5/6-matched (49\%, 95\% CI: 33-62\%) PBSCT ( $p=0.11$; Fig. 4a). GRFS did not 
Fig. 3 Cumulative incidences of acute/chronic GVHD and non-GVHD/relapse mortality. Cumulative incidences of grade III-IV acute GVHD (a), systemically treated chronic GVHD (b), and non-GVHD/ relapse mortality (c) after transplantation from each source. a
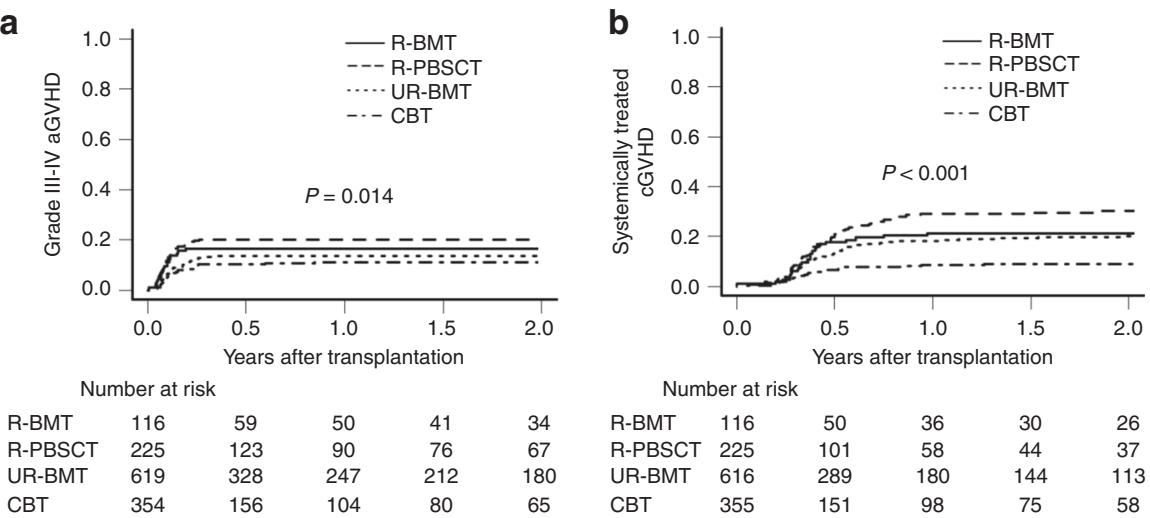
Number at risk

$\begin{array}{lccccc}\text { R-BMT } & 116 & 50 & 36 & 30 & 26 \\ \text { R-PBSCT } & 225 & 101 & 58 & 44 & 37 \\ \text { UR-BMT } & 616 & 289 & 180 & 144 & 113 \\ \text { CBT } & 355 & 151 & 98 & 75 & 58\end{array}$

C

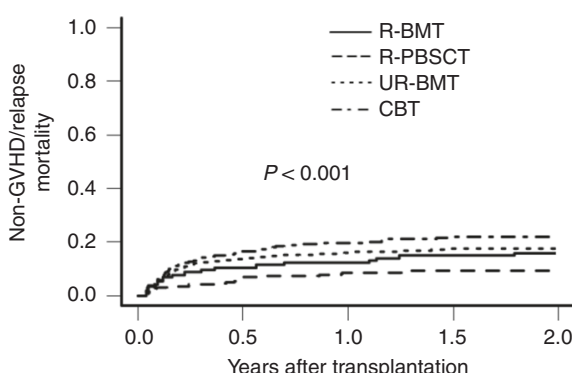
Number at risk

$\begin{array}{lccccc}\text { R-BMT } & 116 & 34 & 29 & 25 & 21 \\ \text { R-PBSCT } & 225 & 63 & 45 & 38 & 34 \\ \text { UR-BMT } & 619 & 215 & 147 & 120 & 98 \\ \text { CBT } & 356 & 102 & 65 & 50 & 39\end{array}$

significantly differ among these three groups (haploidentical: $22 \%, 95 \%$ CI: 10-36\%, 6/6-matched: $20 \%, 95 \%$ CI: 15-26\%, and 5/6-matched: $27 \%$, 95\% CI: $15-40 \%$ at 1 year; $p=0.69$; Fig. 4b). There were no significant differences in the cumulative incidence of relapse after HLA 6/6matched $(40 \%, 95 \%$ CI: $32-47 \%$ at 1 year), HLA 5/6matched (49\%, 95\% CI: 34-63\%), and HLA-haploidentical (52\%, 95\% CI: 34-67\%) PBSCT ( $p=0.33$; Fig. 4c). The cumulative incidences of grade III-IV acute GVHD, systemically-treated chronic GVHD, and NGRM did not differ among the groups (Fig. 4d-f).

\section{Univariate and multivariate analysis of OS and GRFS}

We performed univariate and multivariate analyses of OS and GRFS. In univariate analysis of OS, male sex, higher age ( $>50$ years), worse performance status ( $P S>1$ ), non-CR status at transplantation, and CBT were associated with lower OS (Table S2). Multivariate analysis revealed that male sex, higher age, worse PS, MAC, and non-CR status at transplantation were associated with lower OS, but CBT was not (Table S2). In univariate analysis of GRFS, male sex, higher age, worse PS, non-TBI conditioning, non-CR status at transplantation, and HLA mismatch were associated with lower GRFS (Table 3). Multivariate analysis revealed that male sex, higher age, worse PS, longer duration from diagnosis to transplantation (>180 days), and non-CR status at transplantation were associated with lower GRFS, whereas HLA mismatch was not (Table 3). Donor sources were not associated with GRFS, and the era of with OS or GRFS (Table 3).

\section{Discussion}

The success of allo-HSCT is dependent on suppression of severe complications such as GVHD, relapse, infection, and vital organ failure. However, no one factor can predict longterm survival without ongoing morbidity. GRFS represents ideal recovery from allo-HSCT and is now recognized as an important treatment endpoint. Transplant-treated ATL patients often develop GVHD or other severe complications that lead to limited activity, and thus GRFS is also an important endpoint with respect to QOL. This is the first report of GRFS in ATL, and we have focused on differences in each component associated with GRFS among donor sources. We identified different advantages and disadvantages of each donor source, which provided clues to improve the outcome of allo-HSCT.

We made several important findings regarding CBT. Among patients in $\mathrm{CR}$, OS and relapse rates after CBT were transplantation (2006-10 vs. 2011-15) was not associated 
Table 2 Causes of non-graft-versus-host-disease/relapse deaths.

\begin{tabular}{lllllll}
\hline & R-BMT & R-PBSCT & $\begin{array}{l}\text { UR- } \\
\text { BMT }\end{array}$ & CBT & Haplo & Total \\
\hline Bacterial & 4 & 2 & 17 & 19 & 0 & 42 \\
infection & & & & & & \\
Viral infection & 0 & 0 & 7 & 8 & 0 & 15 \\
Fungal infection & 0 & 0 & 6 & 4 & 1 & 11 \\
Other infection & 1 & 2 & 3 & 5 & 0 & 11 \\
IIP & 2 & 3 & 13 & 7 & 1 & 26 \\
ARDS & 0 & 2 & 5 & 5 & 0 & 12 \\
TMA & 0 & 1 & 3 & 2 & 0 & 6 \\
VOD & 0 & 0 & 5 & 0 & 1 & 6 \\
Bleeding & 1 & 2 & 7 & 3 & 0 & 13 \\
Graft failure & 1 & 0 & 5 & 2 & 0 & 8 \\
Liver failure & 2 & 1 & 4 & 1 & 0 & 8 \\
Heart failure & 1 & 1 & 1 & 0 & 1 & 4 \\
Renal failure & 0 & 3 & 2 & 2 & 0 & 7 \\
CNS failure & 0 & 0 & 2 & 1 & 1 & 4 \\
Lung failure & 1 & 0 & 8 & 2 & 0 & 11 \\
Secondary & 0 & 0 & 3 & 1 & 0 & 4 \\
malignancy & & & 13 & 10 & 1 & 31 \\
Others & 0 & 7 & 104 & 72 & 6 & 219 \\
Total & 13 & 24 & & & & \\
\hline$R-B M T$ & & & & & & \\
\hline
\end{tabular}

$R-B M T$ related bone marrow transplantation, $R-P B S C T$ related peripheral blood stem cell transplantation, $U R-B M T$ unrelated bone marrow transplantation, $C B T$ cord blood transplantation, Haplo: haploidentical transplantation, IIP idiopathic interstitial pneumonia, $A R D S$ acute respiratory distress syndrome, TMA thrombotic microangiopathy, $V O D$ veno-occlusive disease, $C N S$ central nervous system.

not inferior to those after transplantation from the other sources (Fig. 1b, d). GRFS did not significantly differ between patients who underwent CBT and those who underwent transplantation from the other donor sources (Fig. 2a-c). Whereas CBT was associated with a decrease in severe GVHD (Fig. 3a, b), relapse of CR patients after CBT was equivalent to that after transplantation from the other sources (Fig. 1c). These findings imply that the GVL effects of CBT are substantial for CR patients but may be limited in cases with a high tumor burden. On the other hand, the disadvantage of CBT was a high NGRM rate (Fig. 3c), which may be attributable to fatal opportunistic infections in many cases. This derives from delayed immune reconstitution after CBT, which should be further investigated.

In general, GRFS is shorter following PBSCT than BMT due to the higher incidence of chronic GVHD requiring systemic treatment [25]. In our study, however, the duration of GRFS was equivalent in PBSCT and BMT (Fig. 2a-c), due to the low NGRM rate after PBSCT. As described in Table 2, most of the NGRM derived from infectious complications. It is widely recognized that ATL patients often develop severe opportunistic infections [40]. In addition, infection-related mortality of transplant-treated ATL patients is higher than that of patients with other hematological malignancies [26]. PBSCT enables quick recovery of neutrophils and lymphocytes [41], which reduces severe infection. The benefits of reducing infection-related deaths following PBSCT in ATL may be greater than in other hematological malignances. In other words, quick immune reconstitution following PBSCT may be meaningful in ATL, although it remains to be verified in HLA-mismatched settings.

The cumulative incidence of severe GVHD tended to be higher after PBSCT (Fig. 3a, b); however, the relapse rate was not lower after PBSCT than after transplantation from the other donor sources (Fig. 1d, e). On the other hand, the incidence of grade II acute GVHD was higher and the cumulative incidence of relapse was lower after UR-BMT (Fig. 1d, e). These results suggest that moderate GVHD is associated with potent GVL effects and a reduced relapse rate, while severe GVHD is harmful and has no relapse suppression advantages. Many of the patients with severe GVHD possibly died of GVHD itself, infection, or organ failure before the appearance of GVL effects. These findings are compatible with those of another study, which looked at the impact of GVHD on outcomes after alloHSCT for ATL [17].

In analysis of all hematological malignancies in Japan, GRFS was worse after HLA-mismatched transplantation than after HLA-matched transplantation. However, in the present study, HLA matching status did not influence GRFS in multivariate analysis (Table 3). Among patients who underwent PBSCT, the incidence of relapse tended to be higher after HLA-mismatched transplantation (Fig. 4c) and the incidence of grade III-IV acute GVHD tended to be lower after HLA 5/6-matched transplantation (Fig. 4d). This might be because patients who underwent HLAmismatched transplantation received strong GVHD prophylaxis such as ATG, PTCY, and corticosteroids. These results suggest that adjusting the strength of GVHD prophylaxis may improve the outcome of HLA-mismatched transplantation in ATL. The relapse rate was not decreased following HLA-mismatched transplantation; therefore, we could not confirm the HLA-dependent GVL effects in ATL, which should be further explored. On the other hand, the relapse rate tended to be lower after unrelated transplantation (UR-BMT and CBT) than after related transplantation among patients in CR (Fig. 1e). GVL effects may be more potent after unrelated transplantation due to a mechanism other than HLA mismatch, such as mismatch of killer immunoglobulin-like receptor or its ligand polymorphisms [42].

Multivariate analysis revealed that male sex, higher age ( $>50$ years), worse PS $(>1)$, longer duration from diagnosis to transplantation (>180 days), and non-CR status at 
Fig. 4 HLA mismatch influences the outcome of PBSCT. Kaplan-Meier curves of OS (a) and GRFS (b) according to the HLA-matched status. Cumulative incidences of relapse (c), grade III-IV acute GVHD (d), systemically treated chronic GVHD (e), and nonGVHD/relapse mortality (f) according to the HLA-matched status. a

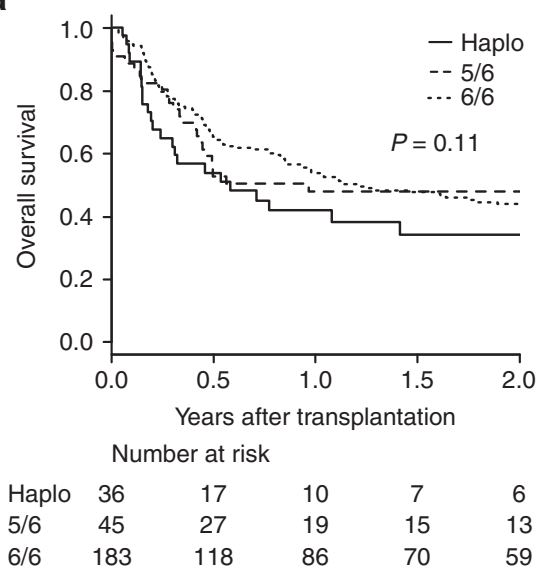

C

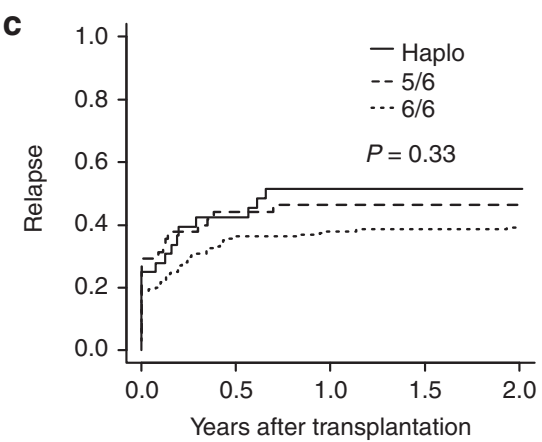

Number at risk

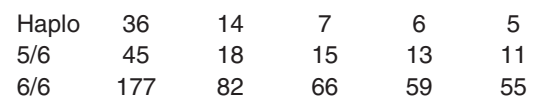

e

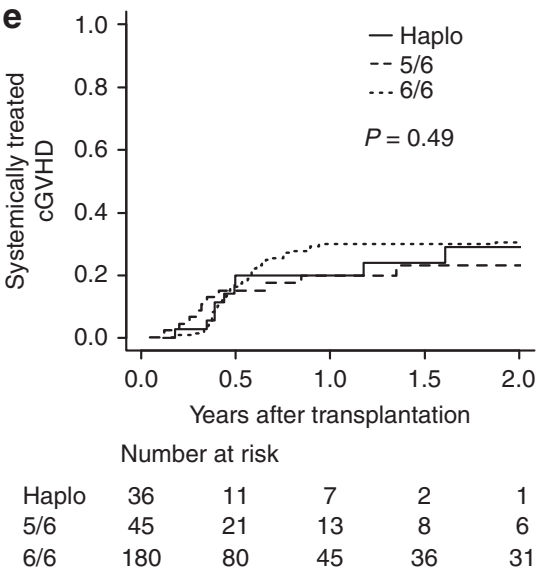

b

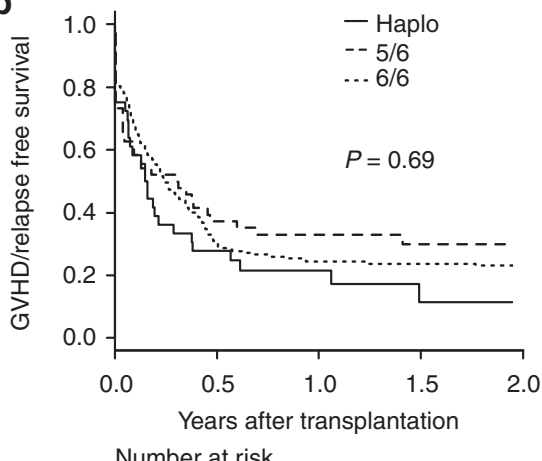

Number at risk

$\begin{array}{lccccc}\text { Haplo } & 36 & 9 & 5 & 2 & 1 \\ 5 / 6 & 45 & 14 & 11 & 8 & 6 \\ 6 / 6 & 180 & 49 & 34 & 30 & 28\end{array}$

d

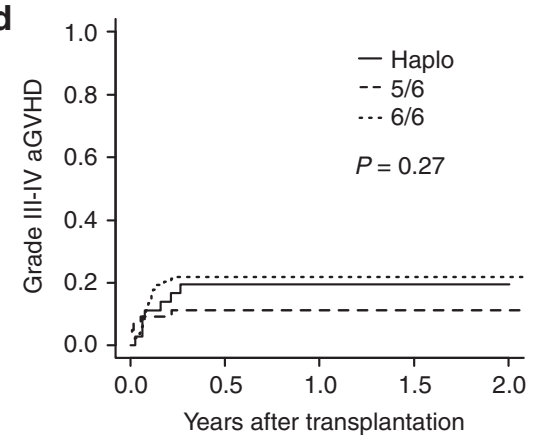
Number at risk

$\begin{array}{lccccc}\text { Haplo } & 36 & 13 & 8 & 5 & 4 \\ 5 / 6 & 45 & 25 & 18 & 15 & 13 \\ 6 / 6 & 180 & 98 & 72 & 61 & 54\end{array}$

f

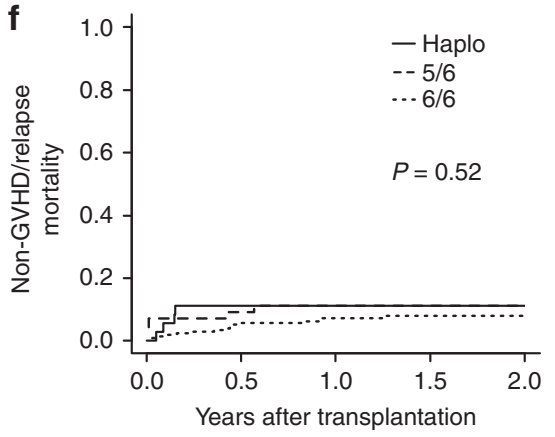
Number at risk

$\begin{array}{lccccc}\text { Haplo } & 36 & 9 & 5 & 2 & 1 \\ 5 / 6 & 45 & 14 & 11 & 8 & 6 \\ 6 / 6 & 180 & 49 & 34 & 30 & 28\end{array}$

transplantation were associated with lower GRFS, which is consistent with the survey of GRFS in all hematological malignancies [25] and the report concerning early donor application for ATL patients [43]. Only 5\% of the patients received ATG; therefore, a larger amount of data must be analyzed to clarify the association between ATG and GRFS in ATL. The outcome of allo-HSCT for non-CR ATL is still unsatisfactory. Many aspects of ATL therapy including conventional chemotherapy, conditioning regimens, GVHD prophylaxis, and maintenance therapies should be further optimized.

This study has inherent limitations that are common among observational studies. Eligibility for transplantation, as well as the choice of transplantation protocol including the selection of graft source, was determined by the treating physicians at each institution. The confounding effect of some variables, such as disease subtype, could not be evaluated because of missing data. Moreover, the data 
Table 3 Univariate and multivariate analyses of graftversus-host disease-free, relapsefree survival.

\begin{tabular}{|c|c|c|c|c|c|c|c|}
\hline \multirow{2}{*}{ Variable $(n)$} & & \multicolumn{3}{|c|}{ Univariate } & \multicolumn{3}{|c|}{ Multivariate } \\
\hline & & HR & $95 \% \mathrm{CI}$ & $P$ & $\mathrm{HR}$ & $95 \% \mathrm{CI}$ & $P$ \\
\hline \multicolumn{8}{|l|}{ Donor source } \\
\hline \multirow[t]{4}{*}{ vs. R-BMT (117) } & R-PBSCT (228) & 1.04 & $0.81-1.34$ & 0.75 & 0.97 & $0.75-1.25$ & 0.80 \\
\hline & UR-BMT (619) & 0.96 & $0.77-1.20$ & 0.74 & 0.97 & $0.75-1.26$ & 0.82 \\
\hline & CBT (359) & 1.15 & $0.91-1.46$ & 0.23 & 1.14 & $0.86-1.52$ & 0.35 \\
\hline & Haplo (40) & 1.31 & $0.88-1.95$ & 0.19 & 1.22 & $0.77-1.95$ & 0.40 \\
\hline \multicolumn{8}{|l|}{ HLA } \\
\hline vs. Matched (716) & Mismatched (647) & 1.13 & $1.00-1.27$ & 0.04 & 1.04 & $0.88-1.22$ & 0.66 \\
\hline \multicolumn{8}{|l|}{ PS } \\
\hline vs. 0-1 (1177) & $2-4(177)$ & 1.83 & $1.55-2.17$ & $<0.001$ & 1.64 & $1.37-1.96$ & $<0.001$ \\
\hline \multicolumn{8}{|l|}{ Disease status } \\
\hline vs. CR (552) & Non-CR (799) & 1.72 & $1.52-1.94$ & $<0.001$ & 1.67 & $1.47-1.90$ & $<0.001$ \\
\hline \multicolumn{8}{|l|}{ Age } \\
\hline vs. $<50$ years (263) & $\geq 50$ years $(1100)$ & 1.30 & $1.11-1.52$ & 0.001 & 1.35 & $1.13-1.60$ & 0.001 \\
\hline \multicolumn{8}{|l|}{ Sex of patients } \\
\hline vs. Female (629) & Male (733) & 1.26 & $1.11-1.42$ & $<0.001$ & 1.27 & $1.12-1.43$ & $<0.001$ \\
\hline \multicolumn{8}{|l|}{ Conditioning } \\
\hline vs. MAC (535) & RIC (822) & 0.98 & $0.87-1.11$ & 0.77 & 0.90 & $0.79-1.03$ & 0.13 \\
\hline vs. no TBI (420) & TBI (940) & 0.86 & $0.76-0.97$ & 0.02 & 0.89 & $0.78-1.02$ & 0.10 \\
\hline \multicolumn{8}{|l|}{ Year } \\
\hline vs. 2006-2010 (600) & 2011-2015 (763) & 1.03 & $0.91-1.16$ & 0.65 & 1.05 & $0.93-1.19$ & 0.45 \\
\hline \multicolumn{8}{|c|}{ Days from diagnosis to transplantation } \\
\hline \multirow[t]{2}{*}{ vs. $<90(84)$} & $90-180(543)$ & 1.07 & $0.82-1.39$ & 0.62 & 1.22 & $0.92-1.60$ & 0.17 \\
\hline & $>180(731)$ & 1.13 & $0.87-1.47$ & 0.35 & 1.35 & $1.02-1.79$ & 0.03 \\
\hline \multicolumn{8}{|l|}{ GVHD prophylaxis } \\
\hline vs. CyA-based (455) & TAC-based (878) & 0.97 & $0.85-1.10$ & 0.63 & 0.96 & $0.82-1.13$ & 0.63 \\
\hline vs. No-ATG (1301) & ATG (62) & 0.93 & $0.70-1.24$ & 0.64 & 0.79 & $0.58-1.09$ & 0.15 \\
\hline
\end{tabular}

$H R$ hazard ratio, $C I$ confidence interval, $R-B M T$ related bone marrow transplantation, $R-P B S C T$ related peripheral blood stem cell transplantation, $U R-B M T$ unrelated bone marrow transplantation, $C B T$ cord blood transplantation, HLA human leukocyte antigen, $P S$ performance status, $C R$ complete remission, MAC myeloablative conditioning, RIC reduced intensity conditioning, TBI total body irradiation, $G V H D$ graftversus-host disease, $C y A$ cyclosporin A, TAC tacrolimus, $A T G$ anti-thymocyte globulin. analyzed did not include information regarding the use of mogamulizumab or lenalidomide, which have both been shown to increase fatal GVHD [44-47].

The present study revealed that transplant from all donor sources is feasible for those patients in CR and that each source can be selected depending on the clinical characteristics of patients. Great efforts should be made to improve or avoid the shortcomings of alternative donor sources, which should ultimately improve the outcome of ATL.

Acknowledgements We thank all the physicians and data managers at the participating institutions who contributed valuable data on transplantation for ATL to the JSHCT. We also thank the members of the data management committees of the JSHCT and the ATL working group for their assistance. The members of the ATL working group are listed below. This work was supported in part by the Practical Research Project for Allergic Diseases and Immunology (Research Technology of Medical Transplantation) from AMED, the Japan
Agency for Medical Research and Development (grant number 19ek0510023h0002). This work was presented as an abstract at the 62nd annual meeting of the American Society of Hematology, Orlando, FL, December 7th, 2019.

The ATL Working Group of the Japanese Society for Hematopoietic Cell Transplantation Makoto Yoshimitsu ${ }^{15}$, Takashi Ishida ${ }^{16}$, Atae Utsunomiya $^{2}$, Koji Kato ${ }^{7}$, Junji Suzumiya ${ }^{17}$, Tomomi Tobai ${ }^{18}$, Koichi Nakase $^{19}$, Yuichiro Nawa ${ }^{19}$, Masakatsu Hishizawa ${ }^{1}$, Takuya Fukushima $^{20}$, Atsushi Wake ${ }^{3}$, Ilseung Choi ${ }^{6}$, Yoshitaka Asakura ${ }^{21}$, Nobuaki Nakano $^{2}$, Hiroshi Fujiwara ${ }^{22}$, Shinichiro Machida ${ }^{23}$, Yasushi Sawayama $^{24}$, Yoshitaka Inoue ${ }^{25}$, Kazunori Imada ${ }^{26}$, Isao Yoshida ${ }^{27}$, Shigeo Fuji ${ }^{28}$, Takahiro Fukuda ${ }^{11}$, Takero Shindo ${ }^{1}$, Masahito Tokunaga $^{2}$, Hiroyuki Muranushi ${ }^{1}$, Satoko Morishima ${ }^{20}$, Shohei Tomori ${ }^{29}$, Tomoki Iemura ${ }^{1}$, Takuya Shimizu ${ }^{1}$, Mari Morita-Fujita ${ }^{1}$.

${ }^{15}$ Department of Hematology and Immunology, Kagoshima University Hospital, Kagoshima, Japan; ${ }^{16}$ Department of Internal Medicine, Division of Hematology and Oncology, Iwate Medical University School of Medicine, Morioka, Japan; ${ }^{17}$ Innovative Cancer Center/ Oncology-Hematology, Shimane University Hospital, Izumo, Japan; 
${ }^{18}$ Department of Internal Medicine III, Division of Hematology and Cell Therapy, Yamagata University Faculty of Medicine, Yamagata, Japan; ${ }^{19}$ Division of Hematology, Ehime Prefectural Central Hospital, Matsuyama, Japan; ${ }^{20}$ Division of Endocrinology, Diabetes and Metabolism, Hematology and Rheumatology, Second Department of Internal Medicine, Graduate School of Medicine, University of the Ryukyus, Nishihara, Japan; ${ }^{21}$ Department of Hematology, Okinawa Red Cross Hospital, Naha, Japan; ${ }^{22}$ Department of Immuno-gene therapy, Center for Comprehensive Cancer Immunotherapy, Mie University Graduate School of Medicine, Tsu, Japan; ${ }^{23}$ Department of Hematology and Oncology, Tokai University School of Medicine, Isehara, Japan; ${ }^{24}$ Department of Hematology, Nagasaki University Hospital, Nagasaki, Japan; ${ }^{25}$ Department of Hematology, Kumamoto University Hospital, Kumamoto, Japan; ${ }^{26}$ Department of Hematology, Osaka Red Cross Hospital, Osaka, Japan; ${ }^{27}$ Department of Hematologic Oncology, Shikoku Cancer Center, Matsuyama, Japan; ${ }^{28}$ Department of Hematology, Osaka International Cancer Center, Osaka, Japan; ${ }^{29}$ Department of Hematology and Oncology, Nakagami Hospital, Okinawa, Japan

Author contributions HM, TS, and KK designed and organized the project, and wrote the paper. HM performed statistical analysis. M Hishizawa, MT, AW, and KK helped to write the paper. NN, TE, M Hidaka, IC, TM, NU, Y Moriuchi, Y Miyazaki, TF, TI, and YA contributed to data gathering. All the authors contributed to the final version of the paper and approved it for publication.

\section{Compliance with ethical standards}

Conflict of interest The authors declare that they have no conflict of interest.

Publisher's note Springer Nature remains neutral with regard to jurisdictional claims in published maps and institutional affiliations.

\section{References}

1. Uchiyama T, Yodoi J, Sagawa K, Takatsuki K, Uchino H. Adult T-cell leukemia: clinical and hematologic features of 16 cases. Blood. 1977;50:481-92.

2. Cook LB, Fuji S, Hermine O, Bazarbachi A, Ramos JC, Ratner L, et al. Revised adult T-cell leukemia-lymphoma international consensus meeting report. J Clin Oncol. 2019;37:677-87. https:// doi.org/10.1200/JCO.18.00501.

3. Tsukasaki K, Fukushima T. JSH practical guidelines for hematological malignancies, 2018: II. lymphoma-8. adult T-cell leukemia-lymphoma. Int J Hematol. 2019;109:249-59. https://doi. org/10.1007/s12185-018-02588-5.

4. Ishitsuka K, Tamura K. Human T-cell leukaemia virus type I and adult T-cell leukaemia-lymphoma. Lancet Oncol. 2014;15: e517-26.

5. Tsukasaki K, Utsunomiya A, Fukuda H, Shibata T, Fukushima T, Takatsuka Y, et al. VCAP-AMP-VECP compared with biweekly CHOP for adult T-cell leukemia-lymphoma: Japan Clinical Oncology Group Study JCOG9801. J Clin Oncol. 2007;25:5458-64. https://doi.org/10.1200/JCO.2007.11.9958.

6. Tsukasaki K, Maeda T, Arimura K, Taguchi J, Fukushima T, Miyazaki Y, et al. Poor outcome of autologous stem cell transplantation for adult $\mathrm{T}$ cell leukemia/lymphoma: a case report and review of the literature. Bone Marrow Transplant. 1999;23:87-9. https://doi.org/10.1038/sj.bmt.1701533.

7. Phillips AA, Willim RD, Savage DG, Horwitz SM, Isola L, Zain $\mathrm{JM}$, et al. A multi-institutional experience of autologous stem cell transplantation in North American patients with human T-cell lymphotropic virus type-1 adult T-cell leukemia/lymphoma suggests ineffective salvage of relapsed patients. Leuk Lymphoma. 2009;50:1039-42. https://doi.org/10.1080/10428190902887571.

8. Ratner L, Rauch D, Abel H, Caruso B, Noy A, Barta SK, et al. Dose-adjusted EPOCH chemotherapy with bortezomib and raltegravir for human T-cell leukemia virus-associated adult T-cell leukemia lymphoma. Blood Cancer J. 2016;6:e408. https://doi. org/10.1038/bcj.2016.21.

9. Ishida T, Jo T, Takemoto S, Suzushima H, Uozumi K, Yamamoto $\mathrm{K}$, et al. Dose-intensified chemotherapy alone or in combination with mogamulizumab in newly diagnosed aggressive adult T-cell leukaemia-lymphoma: a randomized phase II study. Br J Haematol. 2015;169:672-82. https://doi.org/10.1111/bjh.13338.

10. Choi I, Tanosaki R, Uike N, Utsunomiya A, Tomonaga M, Harada $\mathrm{M}$, et al. Long-term outcomes after hematopoietic SCT for adult T-cell leukemia/lymphoma: results of prospective trials. Bone Marrow Transplant. 2011;46:116-8. https://doi.org/10.1038/bmt. 2010.92.

11. Fukushima T, Miyazaki Y, Honda S, Kawano F, Moriuchi Y, Masuda M, et al. Allogeneic hematopoietic stem cell transplantation provides sustained long-term survival for patients with adult T-cell leukemia/lymphoma. Leukemia. 2005;19:829-34.

12. Kami M, Hamaki T, Miyakoshi S, Murashige N, Kanda $\mathrm{Y}$, Tanosaki R, et al. Allogeneic haematopoietic stem cell transplantation for the treatment of adult T-cell leukaemia/lymphoma. Br J Haematol. 2003;120:304-9.

13. Utsunomiya A, Miyazaki Y, Takatsuka Y, Hanada S, Uozumi K, Yashiki $\mathrm{S}$, et al. Improved outcome of adult $\mathrm{T}$ cell leukemia/ lymphoma with allogeneic hematopoietic stem cell transplantation. Bone Marrow Transplant. 2001;27:15-20. https://doi.org/10. 1038/sj.bmt. 1702731.

14. Hishizawa M, Kanda J, Utsunomiya A, Taniguchi S, Eto T, Moriuchi Y, et al. Transplantation of allogeneic hematopoietic stem cells for adult T-cell leukemia: a nationwide retrospective study. Blood. 2010;116:1369-76. https://doi.org/10.1182/blood2009-10-247510.

15. Ishida T, Hishizawa M, Kato K, Tanosaki R, Fukuda T, Taniguchi $\mathrm{S}$, et al. Allogeneic hematopoietic stem cell transplantation for adult T-cell leukemia-lymphoma with special emphasis on preconditioning regimen: a nationwide retrospective study. Blood. 2012;120:1734-41. https://doi.org/10.1182/blood-201203-414490.

16. Bazarbachi A, Cwynarski K, Boumendil A, Finel H, Fields P, Raj $\mathrm{K}$, et al. Outcome of patients with HTLV-1-associated adult T-cell leukemia/lymphoma after SCT: a retrospective study by the EBMT LWP. Bone Marrow Transplant. 2014;49:1266-8. https:// doi.org/10.1038/bmt.2014.143.

17. Kanda J, Hishizawa M, Utsunomiya A, Taniguchi S, Eto T, Moriuchi Y, et al. Impact of graft-versus-host disease on outcomes after allogeneic hematopoietic cell transplantation for adult T-cell leukemia: a retrospective cohort study. Blood. 2012;119:2141-8. https://doi.org/10.1182/blood-2011-07-368233.

18. Ishida T, Hishizawa M, Kato K, Tanosaki R, Fukuda T, Takatsuka $\mathrm{Y}$, et al. Impact of graft-versus-host disease on allogeneic hematopoietic cell transplantation for adult $\mathrm{T}$ cell leukemia-lymphoma focusing on preconditioning regimens: nationwide retrospective study. Biol Blood Marrow Transplant. 2013;19:1731-9. https:// doi.org/10.1016/j.bbmt.2013.09.014.

19. Kato K, Choi I, Wake A, Uike N, Taniguchi S, Moriuchi Y, et al. Treatment of patients with adult $\mathrm{T}$ cell leukemia/lymphoma with cord blood transplantation: a Japanese nationwide retrospective survey. Biol Blood Marrow Transplant. 2014;20:1968-74. https:// doi.org/10.1016/j.bbmt.2014.08.012.

20. Yonekura K, Utsunomiya A, Takatsuka Y, Takeuchi S, Tashiro Y, Kanzaki T, et al. Graft-versus-adult T-cell leukemia/lymphoma effect following allogeneic hematopoietic stem cell 
transplantation. Bone Marrow Transplant. 2008;41:1029-35. https://doi.org/10.1038/bmt.2008.39.

21. Harashima N, Kurihara K, Utsunomiya A, Tanosaki R, Hanabuchi $\mathrm{S}$, Masuda $\mathrm{M}$, et al. Graft-versus-Tax response in adult T-cell leukemia patients after hematopoietic stem cell transplantation. Cancer Res. 2004;64:391-9.

22. Sehn LH, Alyea EP, Weller E, Canning C, Lee S, Ritz J, et al. Comparative outcomes of T-cell-depleted and non-T-cell-depleted allogeneic bone marrow transplantation for chronic myelogenous leukemia: impact of donor lymphocyte infusion. J Clin Oncol. 1999;17:561-8. https://doi.org/10.1200/JCO.1999.17.2.561.

23. Alyea E, Neuberg D, Mauch P, Marcus K, Freedman A, Webb I, et al. Effect of total body irradiation dose escalation on outcome following T-cell-depleted allogeneic bone marrow transplantation. Biol Blood Marrow Transplant. 2002;8:139-44.

24. Holtan SG, DeFor TE, Lazaryan A, Bejanyan N, Arora M, Brunstein CG, et al. Composite end point of graft-versus-host disease-free, relapse-free survival after allogeneic hematopoietic cell transplantation. Blood. 2015;125:1333-8. https://doi.org/10. 1182/blood-2014-10-609032.

25. Inamoto Y, Kimura F, Kanda J, Sugita J, Ikegame K, Nakasone H, et al. Comparison of graft-versus-host disease-free, relapse-free survival according to a variety of graft sources: antithymocyte globulin and single cord blood provide favorable outcomes in some subgroups. Haematologica. 2016;101:1592-602. https://doi. org/10.3324/haematol.2016.149427.

26. Itonaga H, Taguchi J, Fukushima T, Tsushima H, Sato S, Ando K, et al. Distinct clinical features of infectious complications in adult $\mathrm{T}$ cell leukemia/lymphoma patients after allogeneic hematopoietic stem cell transplantation: a retrospective analysis in the Nagasaki transplant group. Biol Blood Marrow Transplant. 2013;19:607-15. https://doi.org/10.1016/j.bbmt.2013.01.011.

27. Fujiwara H, Fuji S, Wake A, Kato K, Takatsuka Y, Fukuda T, et al. Dismal outcome of allogeneic hematopoietic stem cell transplantation for relapsed adult T-cell leukemia/lymphoma, a Japanese nation-wide study. Bone Marrow Transplant. 2017;52:484-8. https://doi.org/10.1038/bmt.2016.313.

28. Uchida N. Cord blood transplantation in Japan. Rinsho Ketsueki. 2016;57:531-6. https://doi.org/10.11406/rinketsu.57.531.

29. Yoshimitsu M, Utsunomiya A, Fuji S, Fujiwara H, Fukuda T, Ogawa $\mathrm{H}$, et al. A retrospective analysis of haplo-identical HLAmismatch hematopoietic transplantation without posttransplantation cyclophosphamide for GVHD prophylaxis in patients with adult T-cell leukemia-lymphoma. Bone Marrow Transplant. 2018. https://doi.org/10.1038/s41409-018-0400-5.

30. Fukushima T, Itonaga H, Moriuchi Y, Yoshida S, Taguchi J, Imaizumi $\mathrm{Y}$, et al. Feasibility of cord blood transplantation in chemosensitive adult T-cell leukemia/lymphoma: a retrospective analysis of the Nagasaki Transplantation Network. Int J Hematol. 2013;97:485-90. https://doi.org/10.1007/s12185-013-1307-y.

31. Nakamura T, Oku E, Nomura K, Morishige S, Takata Y, Seki R, et al. Unrelated cord blood transplantation for patients with adult T-cell leukemia/lymphoma: experience at a single institute. Int $\mathbf{J}$ Hematol. 2012;96:657-63. https://doi.org/10.1007/s12185-0121177-8.

32. Atsuta Y, Suzuki R, Nagamura-Inoue T, Taniguchi S, Takahashi S, Kai S, et al. Disease-specific analyses of unrelated cord blood transplantation compared with unrelated bone marrow transplantation in adult patients with acute leukemia. Blood. 2009;113:1631-8. https://doi.org/10.1182/blood-2008-03-147041.

33. Ballen KK, Gluckman E, Broxmeyer HE. Umbilical cord blood transplantation: the first 25 years and beyond. Blood. 2013;122:491-8. https://doi.org/10.1182/blood-2013-02-453175.
34. Atsuta Y, Suzuki R, Yoshimi A, Gondo H, Tanaka J, Hiraoka A, et al. Unification of hematopoietic stem cell transplantation registries in Japan and establishment of the TRUMP System. Int J Hematol. 2007;86:269-74. https://doi.org/10.1532/IJH97.06239.

35. Atsuta Y. Introduction of Transplant Registry Unified Management Program 2 (TRUMP2): scripts for TRUMP data analyses, part I (variables other than HLA-related data). Int J Hematol. 2016;103:3-10. https://doi.org/10.1007/s12185-015-1894-x.

36. Giralt S, Ballen K, Rizzo D, Bacigalupo A, Horowitz M, Pasquini $\mathrm{M}$, et al. Reduced-intensity conditioning regimen workshop: defining the dose spectrum. Report of a workshop convened by the center for international blood and marrow transplant research. Biol Blood Marrow Transplant. 2009;15:367-9.

37. Bacigalupo A, Ballen K, Rizzo D, Giralt S, Lazarus H, Ho V, et al. Defining the intensity of conditioning regimens: working definitions. Biol Blood Marrow Transplant. 2009;15:1628-33. https://doi.org/10.1016/j.bbmt.2009.07.004.

38. Kanda Y. Investigation of the freely available easy-to-use software 'EZR' for medical statistics. Bone Marrow Transplant. 2013;48:452-8. https://doi.org/10.1038/bmt.2012.244.

39. Kanda J. Scripts for TRUMP data analyses. Part II (HLA-related data): statistical analyses specific for hematopoietic stem cell transplantation. Int J Hematol. 2016;103:11-9. https://doi.org/10. 1007/s12185-015-1907-9.

40. Shimoyama M. Diagnostic criteria and classification of clinical subtypes of adult T-cell leukaemia-lymphoma. A report from the Lymphoma Study Group (1984-87). Br J Haematol. 1991;79:428-37. https://doi.org/10.1111/j.1365-2141.1991.tb08051.x.

41. Pavletic ZS, Joshi SS, Pirruccello SJ, Tarantolo SR, Kollath J, Reed EC, et al. Lymphocyte reconstitution after allogeneic blood stem cell transplantation for hematologic malignancies. Bone Marrow Transplant. 1998;21:33-41. https://doi.org/10.1038/sj. bmt.1701037.

42. Ruggeri L, Capanni M, Urbani E, Perruccio K, Shlomchik WD, Tosti A, et al. Effectiveness of donor natural killer cell alloreactivity in mismatched hematopoietic transplants. Science. 2002;295:2097-100. https://doi.org/10.1126/science.1068440.

43. Fuji S, Fujiwara H, Nakano N, Wake A, Inoue Y, Fukuda T, et al. Early application of related SCT might improve clinical outcome in adult T-cell leukemia/lymphoma. Bone Marrow Transplant. 2016;51:205-11. https://doi.org/10.1038/bmt.2015.265.

44. Fuji S, Inoue Y, Utsunomiya A, Moriuchi Y, Uchimaru K, Choi I, et al. Pretransplantation anti-CCR4 antibody mogamulizumab against adult T-cell leukemia/lymphoma is associated with significantly increased risks of severe and corticosteroid-refractory graft-versus-host disease, nonrelapse mortality, and overall mortality. J Clin Oncol. 2016;34:3426-33. https://doi.org/10.1200/ JCO.2016.67.8250.

45. Fuji S, Shindo T. Friend or foe? Mogamulizumab in allogeneic hematopoietic stem cell transplantation for adult T-cell leukemia/ lymphoma. Stem Cell Investig. 2016;3:70. https://doi.org/10. 21037/sci.2016.09.13.

46. Kneppers E, van der Holt B, Kersten MJ, Zweegman S, Meijer E, Huls $\mathrm{G}$, et al. Lenalidomide maintenance after nonmyeloablative allogeneic stem cell transplantation in multiple myeloma is not feasible: results of the HOVON 76 Trial. Blood. 2011;118:2413-9. https://doi.org/10.1182/blood-2011-04-348292.

47. Coman T, Bachy E, Michallet M, Socié G, Uzunov M, Bourhis $\mathrm{JH}$, et al. Lenalidomide as salvage treatment for multiple myeloma relapsing after allogeneic hematopoietic stem cell transplantation: a report from the French Society of Bone Marrow and Cellular Therapy. Haematologica. 2013;98:776-83. https://doi.org/10. 3324/haematol.2012.069328. 